https://artnodes.uoc.edu

\title{
Neurodiversidad y cognición 4E. Música y multisensorialidad en los entornos Metatopia
}

\author{
Alicia Peñalba \\ Universidad de Valladolid \\ Rubén López-Cano \\ Escuela Superior de Música de Catalunya \\ Jaime del Val \\ Instituto Metabody/Reverso
}

Fecha de presentación: abril de 2021

Fecha de aceptación: mayo de 2021

Fecha de publicación: julio de 2021

\section{Cita recomendada}

Peñalba, Alicia; López-Cano, Rubén; del Val, Jaime. 2021. «Neurodiversidad y cognición 4E. Música y multisensorialidad en los entornos Metatopia». En González Díaz, Paloma; García Méndez, Andrea (coord.). «En los límites de lo posible: arte, ciencia y tecnología». Artnodes, n. ${ }^{0}$ 28: 1-11. UOC. [Fecha de consulta: dd/mm/aa]. http://doi.org/10.7238/a.v0i28.384571

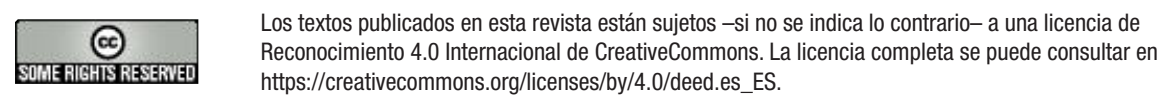

\section{Resumen}

Nuestras sociedades están promoviendo la participación de personas con «discapacidad» 0 neurodiversas en la actividad artística musical. La tecnología, en este sentido, ha desempeñado un importante papel en el desarrollo de dispositivos musicales para la inclusión, que comienzan relacionando gestualidad y sonido pero que evolucionan hacia estructuras multimodales que los combinan con luces, estructuras físicas y arquitectónicas o estímulos hápticos, entre otros. Los entornos Metatopia creados en el contexto del proyecto Metabody han sido diseñados con una concepción que da cabida a la participación de personas neurodiversas. Desde el año 2013 este Metabody colabora con personas con neurodiversidad cognitiva, física y sensorial. Este artículo discute el término «discapacidad» y propone sustituirlo por «neurodiversidad» en consonancia 


\title{
artnodes
}

https://artnodes.uoc.edu

Neurodiversidad y cognición 4E. Música y multisensorialidad en los entornos Metatopia

con las teorías cognitivas del paradigma de la cognición 4E: embodied, embedded, enactive, extended. Se examinan además las posibilidades de Metatopia con personas neurodiversas a través de observaciones y entrevistas. Los resultados de esta investigación apuntan a una expansión de las capacidades de las personas que realizan inmersiones en Metatopia en cuanto a seguridad, iniciativa, ruptura del habitus y concentración. Dichos cambios se explican en términos de las técnicas desarrolladas por del Val en Metatopia: flexinámica, desalineamiento y amorfogénesis y su relación con las teorías de la cognición $4 \mathrm{E}$.

\section{Palabras clave}

neurodiversidad, 4E cognition, inclusión, Metatopia

\section{Neurodiversity and 4E cognition. Music and multisen-soriality in Metatopia environments}

\begin{abstract}
Our societies are promoting the participation of people with a "disability" or neurodiverse people in musical artistic activity. Technology in this sense has played an important role in the development of musical devices for the inclusion, which start to relate to body language and sound but which evolve towards multimodal structures which combine them with lights, physical and architectural structures, haptic stimulants, and other factors. The Metatopia environments created in the context of the project Metabody have been designed with a conception which gives rise to the participation of neurodiverse people. Since 2013 this Metabody has collaborated with people with cognitive, physical and sensorial neurodiversity. This article discusses the term "disability" and proposes replacing it for neurodiversity in concordance with the cognitive theories of the paradigm of $4 E$ cognition: embodied, embedded, enactive, extended. To be examined also are the possibilities of Metatopia with neurodiverse people through observations and interviews. The results of this research point towards an expansion of the capacities of people who carry out immersions in Metatopia regarding security, initiative, the rupture of the hábitus and concentration. Said changes are explained in terms of the techniques developed by the Author in Metatopia: flexinamics, misalignment and amorphogenesis and their relation with the theories of $4 E$ cognition.
\end{abstract}

\section{Keywords}

neurodiversity, 4E cognition, inclusion, Metatopia

\section{Tecnología, música (arte) y «discapacidad»}

En los últimos años, nuestras sociedades promueven la participación en actividades musicales de personas con «discapacidad» 0 con «diversidad funcional» que solían quedar excluidas de ellas. Por medio de diferentes recursos de captación de muy diversos tipos de movimiento, los instrumentos musicales digitales abren nuevas relaciones entre las posibilidades físicas, cognitivas o sensoriales de usuarios singulares y sus posibilidades de generar diversos tipos de música (Wanderley y Depalle 2004). ${ }^{1}$ El SoundBeam, ${ }^{2}$ por ejemplo, emplea sensores de ultrasonido para detectar movimiento. La información recibida produce transfor- maciones musicales proporcionales a la actividad cinética registrada. Ha sido empleado por personas con autismo (Ellis y Leeuwen 2000) 0 personas mayores (Ellis 2004). El MotionComposer también transforma información cinética en sonido y música y fue diseñado específicamente para personas con diversidad funcional (Bergsland y Wechsler 2016). Sus características permiten hacer música y danza sin importar limitaciones físicas o cognitivas (Kontogeorgakopoulos, Wechsler y Keay-Bright 2013) igualando competencialmente a personas neurodiversas y neurotípicas (Peñalba et al. 2019). The Drake Music Project, ${ }^{4}$ por último, ofrece instrumental, recursos y tecnología para dar acceso a la creación musical a personas con «discapacidades» complejas.

1. Algunos instrumentos musicales digitales han sido creados expresamente para favorecer la accesibilidad de cualquier persona independientemente de sus condiciones (Creech 2019) y otros tienen explícitos propósitos terapéuticos (Magee et al. 2018; Partesotti, Peñalba y Manzolli 2018).

2. https://www.soundbeam.co.uk/

3. http://motioncomposer.de/

4. http://www.drakemusicni.com/ 


\section{artnodes}

https://artnodes.uoc.edu

Neurodiversidad y cognición 4E. Música y multisensorialidad en los entornos Metatopia

Desde hace unos años, la tecnología de dispositivos musicales digitales de este tipo está integrando diseños multimodales que no se limitan al movimiento y sonido, sino que incluyen iluminación, dibujo y arquitecturas físicas como parte de una experiencia multimodal. CAREHERE ${ }^{5}$ es un proyecto encaminado a la creación de herramientas para la rehabilitación física de personas con Parkinson y otros trastornos utilizando el dibujo como herramienta y contrastes de sonidos suaves y agresivos (Brooks y Hasselblad 2005). Tenori-on ${ }^{6}$ es un instrumento diseñado por el artista multimedia Toshio Iwai y la marca comercial Yamaha. Se basa en una matriz de 16x16 ledes que permite tocar «música visible» luminosa. Su combinación de información visual y sensorial se ha usado en personas con parálisis cerebral, demencia, daño cerebral, enfermedades crónicas, Parkinson, discapacidad auditiva, autismo y TDAH $^{7}$ entre otras (Clements-Cortes 2014, 62 y 64). MEDIATE ${ }^{8}$ es un proyecto dirigido a niños con autismo que les permite jugar, explorar y ser creativos en un espacio de grandes dimensiones previsible, controlable y seguro que combina aspectos sonoro-musicales, visuales y arquitectónicos (Parés et al. 2005).

Por último, queremos resaltar las instalaciones interactivas Metatopia diseñadas por el artista y filósofo Jaime del Val, creadas en el contexto del proyecto Metabody. ${ }^{9}$ Consisten en un sistema de mallas dinámicas y flexibles sobre las que se proyectan arquitecturas digitales en transformación y movimiento constante y luces y sonido espacializado $0^{10}$ que generan entornos emergentes y multisensoriales (del Val, 2017). Los participantes interactúan con el dispositivo introduciéndose en él y atándose por medio de elásticos a algunas de sus partes y a través de acelerómetros que se adhieren a diferentes partes de su cuerpo. ${ }^{11}$ El principio fundamental es experimentar con movimientos y corporalidades no habituales y promover percepciones novedosas. Del Val lo llama ontohacking o jaqueo de la propia ontología cognitivo-corporal (del Val 2018a). Este se consigue a través de diversas técnicas que pueden ser experimentadas de forma separada 0 integrada. Las técnicas ${ }^{12}$ estudiadas en este artículo son las siguientes (del Val 2020; Peñalba y López-Cano 2018, 135-136):
- La flexinámica ${ }^{13}$ son las estructuras físicas plegables con recubrimiento de red donde se proyecta luz y arquitecturas visuales conectadas a cuerpos humanos que funcionan como extensiones de ellos.

- Los desalineamientos ${ }^{14}$ consisten en incentivar la corporalidad a través de micro-movimientos y microdesplazamientos no habituales informados por nuestro sistema propioceptivo.

- La amorfogénesis ${ }^{15}$ se refiere al diseño de entornos interactivos digitales no cartesianos y no representacionales que producen arquitecturas virtuales que se transforman a partir del movimiento. Propone la emergencia permanente de lo amorfo como «una nueva condición perceptual» y la descentralización de la corporalidad normativa.

A pesar de que estas instalaciones no fueron diseñadas específicamente para ser utilizadas por personas con diversidad funcional han dado muestra de la amplia diversidad cognitiva y motriz de sus participantes. Este artículo analiza las posibilidades de Metatopia en su uso con personas neurodiversas en el período 2013-2020. ${ }^{16}$ Además, la experiencia vivencial de diferentes usuarios con los entornos Metatopia con o sin «habilidades diferentes» obliga a cuestionarse la noción misma de «discapacidad» entendida como una oposición respecto a cierta pretendida «normalidad».

\section{De la "discapacidad" a la neurodiversidad y la cognición corporeizada 4E}

En los últimos diez años el término «discapacidad» está siendo sometido a una exhaustiva revisión. Como alternativa surge la noción de «neurodiversidad», que se refiere a la infinita variación del funcionamiento cognitivo que podemos apreciar en nuestra especie y que ha resultado indispensable para su éxito evolutivo (Mcgee 2012, 12). El concepto fue introducido como una forma de resistencia política

5. Creating Aesthetically Resonant Environments for the Handicapped, Elderly and REhabilitation http://www.bris.ac.uk/carehere/

6. https://www.yamaha.com/en/about/design/synapses/id_005/

7. Trastorno por déficit de atención con o sin hiperactividad.

8. Multisensory environment design for an interface between autistic and typical expressiveness: https://vimeo.com/15387871

9. Véase su sitio web http://metabody.eu/es/

10. La música de los entornos Metatopia consiste en sonidos producidos por síntesis aditiva y frecuencia modulada. No hay pulsaciones rítmicas ni melodías ni cortes o contrastes abruptos. Se trata de continuos texturales espacializados dominados por glissandi de diversas velocidades y longitudes que varían en densidad y color (Peñalba y López-Cano 2018, 141).

11. Para observar la estructura de las instalaciones Metatopia, las dinámicas de interacción e inmersión y el desarrollo de las diferentes técnicas de ontohacking, véase la lista de reproducción de Peñalba y López-Cano (2018): https://www.youtube.com/playlist?list=PLMbKYkVUcdwQSH87qQ4vTjPOjPJL4sVjt.

12. Ver también http://metabody.eu/es/metatopia/

13. http://metabody.eu/es/flexinamica

14. http://metabody.eu/es/desalineamientos

15. http://metabody.eu/es/amorfogenesis

16. De hecho, el Foro Metabody 2019 estaba especialmente orientado a la neurodiversidad. Se pueden consultar todas las colaboraciones en: https://metabody. eu/es/neurodiversidad/ 


\section{artnodes}

https://artnodes.uoc.edu

Neurodiversidad y cognición 4E. Música y multisensorialidad en los entornos Metatopia

por Judy Singer (1999), una socióloga diagnosticada de síndrome de Asperger. De la misma manera que diversos colectivos se reivindican en relación con su clase, género, etnia u orientación sexual (RunswickCole 2014, 1.118), la neurodiversidad es una categoría biopolítica que promueve los derechos de las personas neurológicamente diferentes con respecto a las consideradas como neurotípicas y que intenta prevenir su discriminación (Runswick-Cole 2014, 1.120). Si bien este colectivo reclama que se les reconozca como diferentes, no acepta que se les considere como discapacitados, ${ }^{17}$ un debate que en el contexto español e iberoamericano se ha acrecentado en los últimos años (Rodríguez-Gamero 2020; López-Astorga 2010). El concepto se aplicó primero a personas con trastorno del espectro autista y pronto se extendió su uso a otras condiciones como TDAH, dislexia, trastornos del ánimo, ansiedad, discapacidad intelectual y esquizofrenia (Amstrong 2012, 21).

El discurso de la neurodiversidad tiene muchos puntos de contacto con los principios básicos de teorías cognitivas de tipo ecológico, corporal y enactivo que han inspirado el desarrollo de los entornos Metatopia desarrollados por del Val a los que nos referiremos en este artículo.

El cognitivismo clásico concebía la mente como una entidad separada del cuerpo y la ubicaba exclusivamente en el cerebro. Creía que la percepción capturaba elementos de la realidad exterior para representarlos simbólicamente. La cognición consistía en el proceso computacional de estas representaciones simbólicas. Por el contrario, los enfoques corporeizados defienden la unidad entre mente y cuerpo, la definición mutua entre sujeto y entorno y que la percepción no es la captación pasiva de elementos externos sino una exploración activa, selectiva y corporal. De este modo, la percepción no es independiente del entorno: forman un continuo de interacciones 0 acoples estructurales corporeizados que termina por modular a los sujetos (Varela, Thompson y Rosch 1992; Gibson 1979). Los enfoques corporeizados y enactivos han ido evolucionando para incluir aspectos sociales, emocionales y lingüísticos en torno al paradigma de cognición 4E (Newen, De Bruin y Gallagher 2018). Este paradigma sostiene que la cognición puede ser Embodied, Enactive, Embedded y Extended, es decir que la cognición humana es fundamentalmente encarnada (inseparable de las condiciones corporales y moderada por ellas), actuada (es decir, no solo contemplada sino actuada en el mundo en un continuo entre la vida y la mente), embebida (es decir, situada en el entorno y andamiada por él), y extendida fuera del límite del cráneo para hacer un bucle en el mundo de los recursos extracorporales, tanto materiales como simbólicos (Pohjannoro, 2020, 2). Thomas Amstrong (2012) propone ocho principios que defienden la necesidad de sustituir la noción de «discapacidad» por la de neurodiversidad.
A continuación, los reinterpretaremos bajo la luz de las teorías $4 \mathrm{E}$. Esto nos permitirá comprender mejor las experiencias específicas de personas neurodiversas con la instalación Metatopia.

\section{Principio 1: El cerebro funciona como un ecosistema más que como una máquina (embodied cognition)}

Amstrong propone que es más adecuado hablar de neurodiversidad que de enfermedad o discapacidad pues estos conceptos se fundamentan en aspectos mecanicistas. Es más lógico pensar que el cerebro funciona de forma diversa debido a las características físicas (embodied cognition), contextuales y ambientales de cada persona. La cognición no se ubica exclusivamente en el cerebro porque el cuerpo ha coevolucionado con la mente (Newen, De Bruin y Gallagher 2018) y esta emerge de nuestro historial de interacciones 0 acoplamientos estructurales con el entorno a través de las propiocepciones (Peñalba, 2011).

\section{Principio 2: Los seres humanos y los cerebros humanos existen a lo largo de espectros continuos de competencia (enactive cognition)}

Para las teorías enactivas-ecológicas no tiene por qué haber dos mentes iguales ya que los cuerpos, interacciones y contextos siempre son diferentes (Varela, Thompson y Rosch 1992). De este modo, en lugar de categorías discretas excluyentes expresadas en la oposición binaria «capacidad/discapacidad», es mejor pensar en un continuo donde la eficacia de las competencias y habilidades se definen contextualmente. De hecho, los límites entre «discapacidad» $y$ «normalidad» pueden variar dependiendo del entorno o la tarea a realizar. A fin de cuentas, todos los seres humanos tenemos carencias o limitaciones en algunos ámbitos, tareas o momentos específicos. En este sentido, la competencia se define por el historial de las acciones e interacciones que una persona realiza en un entorno y contexto determinado.

\section{Principio 3: La competencia se define a partir de los valores de la cultura a la que pertenecemos; y Principio 4: El hecho de ser considerado discapacitado o dotado depende en gran medida de cuándo y dónde has nacido (embedded cognition)}

Amstrong defiende que precisamente las capacidades se perciben de forma distinta en función de la cultura en la que estamos inmersos. La neurodiversidad nos ayuda a pensar más en com-

17. El discurso de la neurodiversidad comparte con el «modelo social de la discapacidad» (Oliver 2013; Vázquez-Ferreira 2008) la idea de que las personas con discapacidad pueden contribuir a la sociedad en iguales circunstancias que las demás, desde la valoración a la inclusión y el respeto a lo diverso. 


\section{artnodes}

https://artnodes.uoc.edu

Neurodiversidad y cognición 4E. Música y multisensorialidad en los entornos Metatopia

petencias diferenciadas que en carencias absolutas, pues no en todos los contextos ni en todas las culturas hay acuerdos idénticos en cuanto a lo que se considera «neurotípico». Las concepciones ecológicas de la cognición afirman que esta depende en gran medida del entorno, entendido aquí como un contexto sociocultural determinado. Las diferencias contextuales permiten la puesta en práctica de capacidades diferentes puesto que la cognición no radica en la mente ni en la sociedad ni en la cultura. No en cada uno de estos estamentos aislados; ni siquiera en el conjunto de los tres: emerge en la interacción de los tres.

\section{Principio 5: El éxito de la vida se basa en la adaptación del cerebro a las necesidades del entorno; y Principio 6: El éxito de la vida también depende de la modificación de tu entorno para ajustarlo a las necesidades de tu cerebro único (extended cognition)}

Para desarrollarnos plenamente hemos de, por un lado, adaptarnos a las exigencias del entorno para integrarnos en él; pero al mismo tiempo debemos modificarlo para ajustarlo a nuestras propias necesidades. Si concebimos mente y entorno como dos aspectos diferenciados, este principio puede parecer paradójico o contradictorio. Sin embargo, para las teorías cognitivas $4 \mathrm{E}$ estos procesos no suponen ningún antagonismo puesto que, para ellas, mundo y mente emergen juntos durante el mismo proceso cognitivo (Varela, Rosch y Thompson 1992, 202-206). Por ejemplo, para los filósofos de la mente Clark y Chalmers, usualmente «controlamos nuestro entorno físico y espacial de maneras que alteran fundamentalmente las tareas de procesamiento de información a las que se enfrenta nuestro cerebro» (Clark y Chalmers 1998, 104). La mente descarga en el entorno parte de su trabajo a partir de acciones epistémicas: «una acción cuyo propósito principal es alterar la naturaleza de nuestras propias tareas mentales» y cuyo objetivo es «simplificar o alterar los problemas a los que se enfrentan los cerebros biológicos» (Clark y Chalmers 1998, 105). Estas acciones son, por ejemplo, las operaciones matemáticas que hacemos con la ayuda de nuestros dedos o la escritura en papel, entre otras. Para algunos teóricos de la mente, libretas, ordenadores y teléfonos móviles permiten también que la cognición se expanda por el espacio físico (Rowlands 2009). Los dispositivos musicales digitales que presentamos en la primera parte de este trabajo funcionan también como prótesis extensivas de la mente de sus usuarios: colaboran en que las personas neurodiversas adapten el entorno a sus cerebros únicos.

\section{Principio 7: La construcción de nichos incluye elecciones profesionales y estilos de vida, tecnología de asistencia, recursos humanos, y otras estrategias; y Principio 8: La construcción positiva de nichos modifica el cerebro, que a su vez refuerza la capacidad para adaptarse (extended cognition)}

No todas las personas tenemos las mismas capacidades para el pensamiento abstracto, el desarrollo físico, la organización sistemática 0 la interacción social. Elegir una profesión es también seleccionar un nicho singular adecuado para cada uno de nosotros. Amstrong cree que algunas profesiones pueden favorecer la adaptación de personas con autismo. Por ejemplo, los trabajos que impliquen tareas metódicas 0 que realicen operaciones con algoritmos informáticos. Las tecnologías creativas permiten generar de manera rápida y eficaz nuevos nichos de adaptación para todo tipo de personas.

Como veremos a continuación, del Val plantea la denominada «hipótesis de la plasticidad sensorimotora», que propone movilizar la capacidad de reconfiguración y variación del campo propioceptivo y la integración multisensorial para favorecer la neuroplasticidad y la neurodiversidad, entendida como la capacidad de todo sujeto de desarrollar capacidades cognitivas y motrices en variación continua (del Val 2018b, del Val 2020). A través de su idea de metaterapias, ${ }^{18}$ es decir, la posibilidad de que toda persona amplíe su espectro sensorimotor y con ello afectivo y cognitivo, como parte de una cultura intrínsecamente plural y neurodiversa, los entornos Metatopia funcionan como nichos positivos que permiten a todo tipo de personas expandir su potencial creativo-musical, estético y motor. Los entornos proponen affordances indeterminadas que no imponen un aprendizaje cerrado de movimientos ni una orientación lineal de la percepción, favoreciendo que cada sujeto proyecte en el entorno modos particulares y cambiantes de movimiento propioceptivo e integración multisensorial.

\section{Metatopia, multisensorialidad y nuevos nichos artísticos}

A lo largo de todo el proyecto Metabody, la idea de metaterapias y la colaboración con personas neurodiversas ha sido constante (ver imagen 1).

Para esta investigación se llevaron a cabo observaciones in situ y a través de vídeo; entrevistas a los participantes y educadores que los acompañaban ${ }^{19}$ y entrevistas al coordinador del proyecto Metatopia Jaime del Val (ver imagen 2). En el análisis de la información obtenida de la codificación abierta emergieron algunas categorías que

18. www.metabody.eu/es/metaterapias

19. Las observaciones in situ se realizaron en el Foro Metaboby realizado en Medialab Prado los días 26 y 27 de julio de 2019. 


\section{artnodes}

\begin{tabular}{|c|l|l|l|}
\hline Fecha & \multicolumn{1}{|c|}{ Lugar } & \multicolumn{1}{|c|}{ Colectivo o persona } & \multicolumn{1}{|c|}{ Neurodiversidad } \\
\hline 2013 & Madrid & $\begin{array}{l}\text { Marta Leirado y varios } \\
\text { performers }\end{array}$ & Neurodiversidad física \\
\hline $\begin{array}{c}2013- \\
2016\end{array}$ & $\begin{array}{l}\text { Madrid, Génova, } \\
\text { Dresden, Estambul, } \\
\text { Weimar, Berkeley, Paris }\end{array}$ & Palindrome & Diversos colectivos \\
\hline 2014 & Madrid & $\begin{array}{l}\text { Colectivos invitados por } \\
\text { Palindrome }\end{array}$ & $\begin{array}{l}\text { Diversos colectivos y personas con } \\
\text { parálisis cerebral }\end{array}$ \\
\hline 2014 & Estambul & $\begin{array}{l}\text { Colectivos invitados por } \\
\text { Palindrome }\end{array}$ & $\begin{array}{l}\text { Síndrome Down y neurodiversidad } \\
\text { sensorimotora y cognitiva }\end{array}$ \\
\hline 2014 & Amsterdam & Van Boijen Institute & Diversos colectivos \\
\hline 2015 & Berkeley, California & $\begin{array}{l}\text { Neil Marcus y activistas- } \\
\text { artistas neurodiversos }\end{array}$ & Personas con parálisis cerebral \\
\hline 2015 & Weimar & $\begin{array}{l}\text { Petra Kuppers, activistay y } \\
\text { teórica de la discapacidad }\end{array}$ & Neurodiversidad física \\
\hline 2015 & Weimar & $\begin{array}{l}\text { Colectivos invitados por } \\
\text { Palindrome }\end{array}$ & $\begin{array}{l}\text { Síndrome Down y neurodiversidad } \\
\text { sensorimotora y cognitiva }\end{array}$ \\
\hline 2016 & Londres & Colaboración independiente & Personas con discapacidad visual \\
\hline 2016 & Madrid & Psico-ballet de Maite León & Personas con Síndrome de Down \\
\hline 2016 & Madrid & $\begin{array}{l}\text { Colaboración independiente } \\
\text { vía Marta Leirado }\end{array}$ & Personas con parálisis cerebral \\
\hline 2017 & Madrid & $\begin{array}{l}\text { APAM. Asociación via Marta } \\
\text { Leirado }\end{array}$ & Personas con parálisis cerebral \\
\hline 2017 & Madrid & Mónica Lamberti. Artista & Osteogénesis imperfecta \\
\hline 2018 & Madrid & $\begin{array}{l}\text { Soledad Arnau. Activistay } \\
\text { filósofa }\end{array}$ & Tetraplejia \\
\hline 2018 & $\begin{array}{l}\text { Peñaranda de } \\
\text { Bracamonte } \\
\text { (Salamanca) }\end{array}$ & ACOPEDIS, Asociación & $\begin{array}{l}\text { Personas diversas con TEA, parálisis } \\
\text { cerebral, Síndrome de Down }\end{array}$ \\
\hline 2019 & Madrid & AMP Pinto. Asociación & $\begin{array}{l}\text { Personas con neurodiversidad intelectual, } \\
\text { TEA, parálisis cerebral, Síndrome de Down }\end{array}$ \\
\hline 2019 & $\begin{array}{l}\text { La Haya, campo de } \\
\text { refugiados de Rijswijk }\end{array}$ & Thomas Tajo, activista & Persona con discapacidad visual \\
\hline 2020 & Madrid Bruselas & $\begin{array}{l}\text { Thomas Tajo, activista } \\
\text { Visioninclusive.org }\end{array}$ & Persona con discapacidad visual \\
\hline
\end{tabular}

Imagen 1. Colaboraciones del Proyecto Metabody con personas, colectivos y proyectos relacionados con neurodiversidad (2013-2020)

apuntan a que las diversas técnicas empleadas en las instalaciones Metatopia contribuyen a convertirlas en nichos positivos que permiten la expansión de las capacidades físicas, cognitivas o emocionales de las personas neurodiversas. Las categorías que detectamos son: sentimiento de seguridad y descanso, iniciativa, ruptura del habitus y concentración.

\section{Sentimiento de seguridad y descanso}

Muchas personas neurodiversas suelen mostrarse inseguras ante situaciones nuevas. Además, las estructuras Metatopia se yerguen en salas oscuras y poseen paneles flexibles de varios metros de alto, luces interactivas y música envolvente. Esto crea una sensación inicial de «agresividad». Al respecto, la educadora de Esmeralda ${ }^{20}$ nos contó que esta «al principio no quería entrar por miedo» pero que este se disipó una vez dentro de la instalación. Por su parte, la educadora que hace la entrevista con Rebeca comenta su reparo inicial de que esta entrara en la instalación pues con frecuencia «padece crisis epilépticas». Rebeca «tiene mucho miedo, en general», y siempre se muestra reticente a «participar en las actividades que hacen en el centro». Sin embargo, su actitud la sorprendió. Durante su experiencia «la he visto muy tranquila y disfrutando, no la he visto con miedo en absoluto».

\begin{tabular}{|c|l|l|}
\hline \multirow{4}{*}{ Participantes } & $\begin{array}{l}\text { Personas con } \\
\text { neurodiversidad }\end{array}$ & Especificado en tabla 1 \\
\cline { 2 - 3 } & Educadores & $\begin{array}{l}\text { 4 Personas acompañantes de las asociaciones } \\
\text { (mediadores en las entrevistas y experimentaciones) }\end{array}$ \\
\cline { 2 - 4 } & Investigadores & $\begin{array}{l}\text { 2 observadores no participantes } \\
\text { Coordinador del proyecto, observador participante }\end{array}$ \\
\hline \multirow{4}{*}{$\begin{array}{c}\text { Técnicas de } \\
\text { recogida de } \\
\text { datos }\end{array}$} & $\begin{array}{l}\text { Observación } \\
\text { in situ }\end{array}$ & $\begin{array}{l}\text { 6 personas AMP Pinto (Madrid. Asociación sin ánimo de } \\
\text { lucro. Personas con neurodiversidad intelectual) } \\
8 \text { personas Fundación Gil Gayarre (Pozuelo de Alarcón. } \\
\text { Personas con neurodiversidad intelectual) }\end{array}$ \\
\cline { 2 - 3 } & $\begin{array}{l}\text { Observaciones a } \\
\text { través de vídeo }\end{array}$ & Personas de otras asociaciones y colectivos (ver tabla 1) \\
\cline { 2 - 3 } & Entrevistas & $\begin{array}{l}\text { 14 entrevistas a educadores } \\
\text { 2 entrevistas al coordinador del proyecto }\end{array}$ \\
\hline $\begin{array}{c}\text { Técnicas de } \\
\text { análisis de } \\
\text { datos }\end{array}$ & $\begin{array}{l}\text { Transcripción } \\
\text { observaciones y } \\
\text { entrevistas }\end{array}$ & $\begin{array}{l}\text { Codificación Axial } \\
\text { Reducción de categorías }\end{array}$ \\
\hline
\end{tabular}

Imagen 2. Metodología. Participantes, técnicas de recogida y análisis de datos.

Montse es performer y padece ontogénesis imperfecta 0 «enfermedad de huesos de cristal»; una falta de colágeno congénita que hace los huesos extremadamente frágiles. Tareas simples como caminar le produce mucha inseguridad puesto que «cualquier caída podría romperle varios huesos y tener complicaciones». Al principio tuvo reparos en participar en la experiencia pues el sonido de la instalación le parecía «muy estridente», y comentó: «me puedo caer». Finalmente realizó la inmersión, durante la cual se sintió «increíblemente segura». Declaró que ella solo se siente así «en el agua, y allí dentro me he sentido como en el mar».

Una sensación muy parecida tuvo Laura, persona neurodiversa intelectualmente y con dificultades en el movimiento. La educadora que viene con ella resalta la seguridad que mostró durante su inmersión en la instalación: «últimamente siente mucho miedo al caminar, tiene miedo a caerse y ahí dentro la he visto muy segura». A Lidia, una persona neurodiversa con dificultades físicas «le cuesta mucho caminar, se cansa mucho». Sin embargo, pese a que se le puso una silla dentro de la instalación para que pudiera descansar o apoyarse, la educadora resaltó que, a lo largo de su inmersión, en todo momento «ha estado sola y de pie todo el tiempo, no ha necesitado sentarse... [tuvo] mucha sensación de seguridad... hasta ha saltado».

Entre las diversas técnicas de Metatopia que colaboran a esta sensación de seguridad destaca la flexinámica. Al atarse a la estructura de mallas los sujetos sienten apoyo y soporte a sus movimientos (imagen 3). Cada uno de sus gestos se ve expandido y distribuido por toda la estructura: el movimiento no solo depende de ellos sino de todo el dispositivo, lo que produce cierta sensación de «acompañamiento». Los sujetos viven el proceso como una extensión de su propio cuerpo tanto al nivel del impulso efector de movimiento como en el de la percepción. La estructura guía y acompaña todo el proceso favoreciendo una gestualidad

20. Todos los nombres propios que aparecen a continuación son seudónimos. 


\section{artnodes}

https://artnodes.uoc.edu

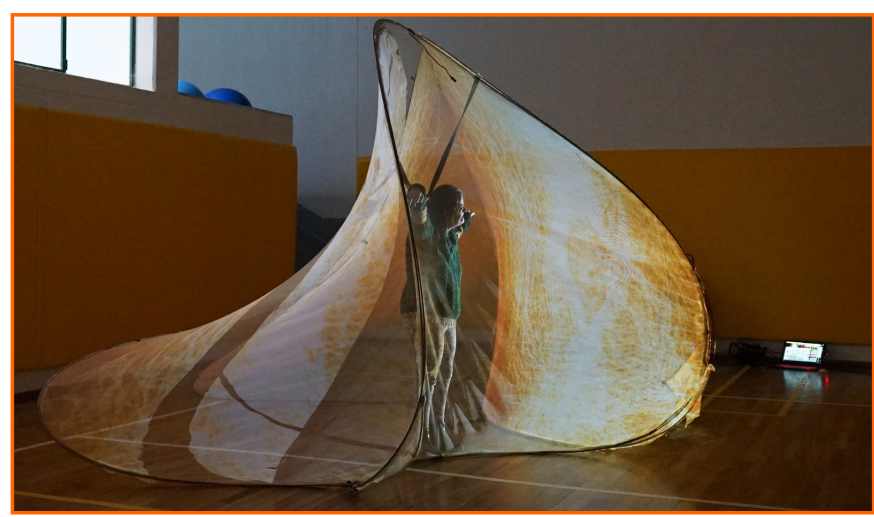

Imagen 3. Persona neurodiversa en una inmersión (Madrid, Fundación Gil Gayarre).

fluida que les permite gestionar mejor la diversidad e intensidad de estímulos audiovisuales que reciben. Las mallas transparentes evitan la claustrofobia, dando la sensación tanto de contención como de libertad.

\section{Iniciativa}

Muchas personas neurodiversas suelen tener dificultades para mostrar iniciativa en contextos no conocidos. En palabras de una de las educadoras, «suelen necesitar apoyo y ayuda para casi todo, les cuesta mucho ser creativos y proponer cosas nuevas». Del Val nos comentó que en un taller realizado con la compañía de ballet de personas con neurodiversidad intelectual de Maite León «los profesores creían que había que darles una guía y un patrón de imitación a los participantes». Sin embargo, su ejercicio de inmersión demostró que estos eran capaces de construir sus propios recorridos y exploraciones motoras. En otro taller, del Val interactuaba fuera de la instalación para motivar a las personas que estaban dentro. En ocasiones estas se mostraban extremadamente pasivas. A veces se limitaban a imitarlo. Pero en otros momentos el artista propuso a los sujetos tomar la iniciativa y ser él quien les siguiera.

La baja iniciativa que se atribuye a las personas neurodiversas no necesariamente forma parte de su condición. Es un resultado de las circunstancias contextuales. Con frecuencia sus acciones corren el riesgo de ser juzgadas como erróneas o incorrectas en relación con las personas neurotípicas. Esto propicia que se retraigan. La programación a través de la técnica de amorfogénesis deshace cualquier posibilidad de juicio en la instalación. No hay reglas, no hay un sistema de valores previos, no hay una manera de interactuar bien o mal con el dispositivo pues este no responde a patrones de control habitual y de planificación intelectual. Con ello se potencian otro tipo de capacidades como la intuición, la escucha corporal y la capacidad de cocreación con las estructuras. El éxito interactivo depende de la puesta en práctica de estas capacidades que no son exclusivas de las personas neurotípicas, con lo que las personas neurodiversas se sienten empoderadas y ejercen su creatividad e iniciativa.

\section{Ruptura del habitus}

En Peñalba y López-Cano (2018) estudiamos cómo la interacción con las instalaciones Metatopia invita a romper habitus, entendidos estos como el «sistema de disposiciones durables y transportables que, integrando todas las experiencias pasadas, funciona en cada momento como una matriz de percepciones, de apreciaciones y de acciones» gracias a las cuales desarrollamos varias tareas cotidianas (Bourdieu 2012). En las inmersiones con la instalación, tanto personas neurodiversas como neurotípicas logran trascender sus habitus y descubrir nuevas posibilidades de movimiento, percepción y acción en el entorno (imagen 4). La educadora comenta que Laura «es una persona que no mueve mucho los brazos, es bastante pasiva». Sin embargo, dentro de la instalación «la he visto experimentar mucho con los brazos y me ha sorprendido mucho». Por el contrario, David, que tiene un diagnóstico de TDAH, nos cuenta la educadora, en «general no para de moverse» todo el tiempo. Sin embargo, durante su inmersión su hiperactividad se apaciguó y se le vio «mucho más quieto de lo habitual [...]. Mover los brazos estando con los pies parados es significativo, yo no le había visto nunca así [.. . ]. Se le veía disfrutar mucho. Había momentos de conexión». Esther, por su parte, hizo «movimientos con mucha energía y eso no suele ser habitual» en ella. Por último, Rebeca experimentó con «nuevos movimientos».

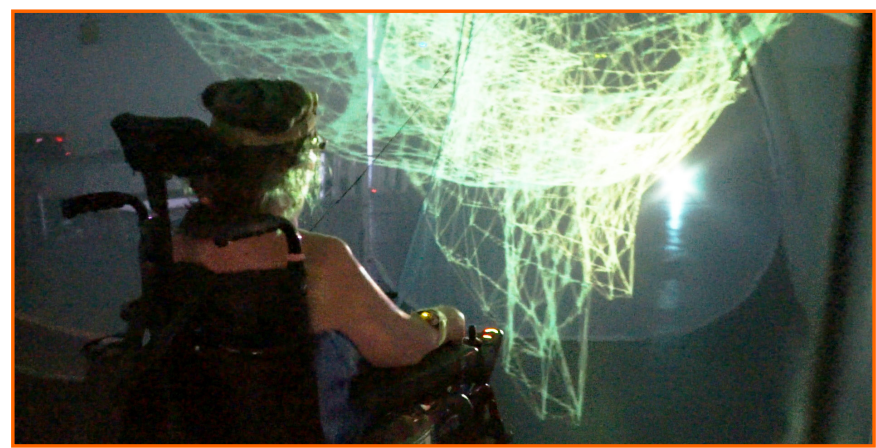

Imagen 4. Persona con neurodiversidad física en una inmersión.

Las personas neurodiversas tienen patrones y rutinas gestuales también diversas. La técnica de desalineamientos empleada en las inmersiones hace que la gestualidad diversa no sea inhibida sino potenciada. Las estructuras físicas y la forma de agarre en puntos del cuerpo no vinculados con el control manual facilitan la exploración de gestualidades diversas, una gestualidad que surge en cocreación con el dispositivo y que retroalimenta una serie de competencias que no suelen ser visibilizadas en la cotidianidad. Además, el mapeado que correlaciona los movimientos con las respuestas de la instalación, que en este caso es no causal, invita a no reproducir relaciones causa-efecto habituales. Esto, aunado al sentimiento de seguridad y al impulso de iniciativa, permite que los sujetos se atrevan a romper con lo conocido. 


\section{artnodes}

\section{Concentración}

Existen muchas formas de mantener la atención y concentrarse. № obstante, con frecuencia se señala la dificultad de concentración de las personas neurodiversas. En las inmersiones observadas detectamos diversos tipos de concentración en algunos cambios de su conducta habitual. Lorenzo suele verbalizar mucho, pero, en su ejercicio de inmersión, moderó bastante el uso del lenguaje. Por su parte, David y Juan, ambos diagnosticados con TDAH, redujeron su habitual hiperactividad hasta llegar a la quietud absoluta. En relación con un taller donde realizaron inmersión personas con parálisis cerebral, del Val afirma que algunas de estas «pasaron varias horas en la instalación, no querían salir. Según la mediadora se mostraron extraordinariamente activos y concentrados». Para él, «estaban descubriendo aspectos que en otros contextos no se daban». De este modo, su exploración del entorno Metatopia les condujo a descubrir formas de interacción «táctiles 0 propioceptivas» que no encontraban en otras situaciones.

La amorfogénesis invita a una percepción de lo amorfo a través del sistema de estructuras físicas, luces y sonidos que producen estímulos constantemente cambiantes que captan continuamente la atención de los sujetos. Por otro lado, al ser un entorno que se crea al mismo tiempo que es explorado perceptualmente por los participantes, construye un nicho autónomo que funciona completamente distinto al mundo habitual, generando nuevas formas de atención, percepción y concentración. Muchos sujetos se sienten extraídos del mundo cotidiano y sus distracciones. Durante los ejercicios la atención se puede focalizar en diversos aspectos: cuerpo, movimiento, estructuras físicas, luces y sonidos, o puede ser difusa y amorfa, propiciando formas de atención diferentes. De este modo, si se cumplen las condiciones de seguridad iniciativa y ruptura del habitus es muy frecuente descubrir momentos de absoluta concentración, bien porque se tienen experiencias nunca antes experimentadas (motrices, táctiles, visuales, auditivas), o bien por la abstracción del mundo cotidiano.

\section{Conclusiones}

Las instalaciones Metatopia ofrecen nichos particulares donde tanto personas neurodiversas como neurotípicas descubren maneras diferentes de interactuar con el entorno, facilitando experiencias que jaquean sus habitus cognitivos corporales y promoviendo su neuroplasticidad como forma de terapia (metaterapias). En el caso de personas neurodiversas, este nicho les permite interactuar en iguales circunstancias que a otras personas, en línea con las ideas del «modelo social de la discapacidad». Son capaces de desplegar acciones corpóreo-cognitivas que en los entornos físico-culturales habituales les resultan inaccesibles. Esto es posible porque en estos nichos el entorno es percibido y explorado al mismo tiempo en que se crea, lo cual invita a que la experiencia de los participantes sea corporeizada, embebida, enactiva y extendida, en sintonía con el paradigma de la cognición 4E. Esta experiencia es encarnada en el sentido de que es inseparable de las condiciones corporales y modelada por ellas. Incentiva las gestualidades diversas y fomenta romper con el habitus. La experiencia es actuada, es decir, no solo contemplada sino actuada en el mundo en un continuo entre la vida y la mente, generando una potente iniciativa por parte del participante. Es embebida, es decir, situada en el entorno y andamiada por él, un entorno que produce seguridad y que promueve la creación de un mundo diverso y constantemente cambiante que favorece la concentración. Y, por último, es extendida fuera del límite del cráneo, al cuerpo, e incluso a las propias estructuras físicas, que se convierten en extensiones de la persona, favoreciendo la seguridad a través de la posibilidad de una cocreación estética constantemente acompañada.

\section{Referencias bibliográficas}

Amstrong, Thomas. El poder de la neurodiversidad. Barcelona: Paidós, 2012.

Bergsland, Andreas y Robert Wechsler. «Turning movement into music». Soundeffects. An Interdisciplinary Journal of Sound and Sound Experience 6, n. ${ }^{0} 1$ (2016): 24-47. DOI: https://doi. org/10.7146/se.v6i1.24911.

Bourdieu, Pierre. Bosquejo de una teoría de la práctica. Buenos Aires: Prometeo, 2012.

Brooks, Anthony Lewis y Stefan Hasselblad. «Creating aesthetically resonant environments for the handicapped, elderly and rehabilitation: Sweden». International Journal on Disability and Human Development 4, n. ${ }^{\circ} 4$ (2005): 285-294. 10.1515/IJDHD.2005.4.4.285.

Clark, Andy y David Chalmers. «The extended mind». Analysis 58, n. ${ }^{0} 1$ (1998): 7-19. DOI: https://doi.org/10.1093/analys/58.1.7.

Clements-Cortes, Amy. «Getting your groove on with the Tenori-0n». Journal of Music, Technology \& Education 7, n. ${ }^{0} 1$ (2014): 59-74. DOI: https://doi.org/10.1386/jmte.7.1.59_1.

Creech, Andrea. «Using music technology creatively to Enrich laterlife: A literature review». Frontiers in Psychology 10 (2019). DOl: https://doi.org/10.3389/fpsyg.2019.00117.

del Val, Jaime. «Neither Human nor Cyborg: I Am a Bitch and a Molecular Swarm. Pro-prioception, Body Intelligence and Microsexual Conviviality». World Futures, 76 (2020):5-7, pp. 314-336. D0I: 1 https://doi.0.1080/02604027.2020.1778335.

del Val, Jaime. «Ontohacking: Onto-Ecological Politics in the Algoricene». Leonardo, 51:2 (2018a): 187-188. D0I: 10.1162/ LEON_a_01521.

del Val, Jaime. «Microsingularities: Body Intelligence, Neuroplastic Ecologies and Antismart Architectures in the Algoricene». In Balance-Unbalance Conference (2018a) Proceedings, pages 


\section{artnodes}

65-69. Anne Nitgen Ed. The Patching Zone, Rotterdam.- www. reverso.org/texts/DelVal-2018-Microsingularities--BunB2018.pdf

Ellis, Phil. «Improving quality of life and well-being for children and the elderly through vibroacoustic sound therapy». En Computers helping people with special needs, editado por Klaus Miesenberger, Joachim Klaus, Wolfgang L. Zagler y Dominique Burger, 416-422. Londres: Springer, 2004.

Ellis, Phil y Lieselotte van Leeuwen. «Living sound: Human interaction and children with autism». ISME Commission on Music in Special Education, Music Therapy and Music Medicine (2000): 1-23. http://www.soundbeam.it/autism.pdf.

Gibson, James J. The ecological approach to visual perception. Nueva Jersey: Lawrence Erlbaum, 1979.

Kontogeorgakopoulos, Alexandros, Robert Wechsler y Wendy KeayBright. «Camera-based motion tracking and performing arts for persons with motor disabilities and autism». En Assistive technologies, disability informatics and computer access for motor limitations, editado por Georgios Kouroupetroglou, 294-322.Pensilvania, Hershey PA, USA: Information Science Reference Press (IGI Global), 2013 . DOI: https://doi.org/10.4018/978-1-4666-4442-7.

López-Astorga, Miguel. «Neurodiversidad y razonamiento lógico». Revista Educación Inclusiva 3, n. ${ }^{\circ} 2$ (2010): 97-111. https:// revistaeducacioninclusiva.es/index.php/REl/article/view/207.

Magee, W. L., M. Bertolami, L. Kubicek, M. LaJoie, L. Martino, A. Sankowski, J. Townsend, A. M. Whitehead-Pleaux y J. B. Zigo. «Using music technology in music therapy with populations across the life span in medical and educational programs". Music and Medicine 3, n. ${ }^{\circ} 3$ (2011): 146-153. DOl: https://doi. org/10.1177/1943862111403005.

Mcgee, Micki. «Neurodiversity». Contexts 11, n. ${ }^{\circ} 3$ (2012): 12-13. DOI: https://doi.org/10.1177/1536504212456175.

Newen, Albert, Leon De Bruin y Shaun Gallagher. «4E Cognition: Historical roots, key concepts, and central issues». En The Oxford Handbook of 4E Cognition, 3-18, editado por Albert Newen, Leon De Bruin y Shaun Gallagher. Oxford: Oxford University Press, 2018. D0I: https://doi.org/10.1093/oxfordhb/9780198735410.001.0001.

Oliver, Mike. «The social model of disability: Thirty years on». Disability \& Society 28, n. 7 (2013): 1.024-1.026. D0I: https://doi.org/10. 1080/09687599.2013.818773.

Partesotti, Elena, Alicia Peñalba, and Jônatas Manzolli. «Digital Instruments and Their Uses in Music Therapy». Nordic Journal of Music Therapy, 27 n. ${ }^{0}$ 5, (2018): 399-418. https://doi.org/10.10 80/08098131.2018.1490919.

Pohjannoro, Ulla. «Embodiment in composition: 4E theoretical considerations and empirical evidence from a case study». Musicae Scientiae (2020): 1-18. https://doi.org/10.1177/1029864920961447.
Parés, Narcís, P. Freixa, J. L. Ribas, A. Carreras, J. Durany, J. Ferrer, D. Gomez et al. «Estrategias de comunicación interactiva en un espacio multisensorial para niños y niñas con autismo profundo". Formats. Revista de Comunicació Audivisual Audivisual 4 (2005): 1-6. https://raco.cat/index.php/Formats/article/view/257330.

Peñalba,Alicia. «Towards a theory of proprioception as a bodily basis for consciousness in music». Clarke, D. and Clarke, E. (eds) Music and consciousness: Philosophical, psychological, and cultural perspectives. Oxford: Oxford University Press. (2011): 215-231. doi: https://doi.org/10.1093/acprof:0so/9780199553792.003.0066.

Peñalba, Alicia y López-Cano, Rubén. «Hackeo ontológico y entornos Metatopia: Performance motora, sistemas interactivos y experiencia subjetiva». Resonancias: Revista de Investigación Musical 4, n. ${ }^{0}$ 43 (2018): 133-157. DOI: https://doi.org/10.7764/res.2018.43.7.

Peñalba, Alicia, María-José Valles, Elena Partesotti, María-Ángeles Sevillano, and Rosario Castañón. «Accessibility and Participation in the Use of an Inclusive Musical Instrument: The Case of MotionComposer». Journal of Music, Technology and Education 12, n. ${ }^{0}$ (2019): 79-94. https://doi.org/10.1386/jmte.12.1.79_1.

Rodríguez-Gamero, Marco Alonso. «Nuevas perspectivas conceptuales en la afirmación del derecho a la igualdad de las personas con discapacidad mental: una evaluación crítica de la jurisprudencia del Tribunal Constitucional peruano». Estudios Constitucionales 18, n. ${ }^{0} 1$ (2020): 143-210. DOI: https://doi.org/10.4067/S071852002020000100143.

Rowlands, Mark. «The extended mind». Zygon 44, n. ${ }^{\circ} 3$ (2009): 628641. D0I: https://doi.org/10.1111/j.1467-9744.2009.01021.x.

Runswick-Cole, Katherine. "Us" and "them": The limits and possibilities of a "politics of neurodiversity" in neoliberal times». Disability \& Society 29, n. 7 (2014): 1.117-1.129. DOI: https://doi.org/10 $.1080 / 09687599.2014 .910107$.

Singer, Judy. «Why can't you be normal for once in your life? From a problem with no name to the emergence of a new category of difference». Disability discourse (1999): 59-70. https://www.divaportal.org/smash/get/diva2:457919/FULLTEXT01.pdf-title=Autism.

Varela, Francisco J., Evan Thompson y Eleanor Rosch. En De cuerpo presente: las ciencias cognitivas y la experiencia humana. Barcelona: Gedisa, 1992.

Vázquez-Ferreira, Miguel. «La construcción social de la discapacidad: Habitus, estereotipos y exclusión social». Nómadas. Revista Crítica de Ciencias Sociales y Jurídicas 17, n. ${ }^{0} 1$ (2008): 221-232. http://revistas.ucm.es/index.php/NOMA/article/view/ NOMA0808120221A.

Wanderley, Marcelo M. y Philippe Depalle. «Gestural control of sound synthesis». Proceedings of the IEEE 92, n. ${ }^{\circ} 4$ (2004): 632-644. DOI: https://doi.org/10.1109/JPROC.2004.825882. 


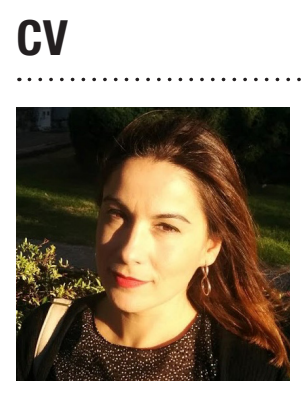

\section{Alicia Peñalba}

Universidad de Valladolid

alicia.penalba@uva.es

Profesora titular de música (ac. catedrática) en la Universidad de Valladolid. Licenciada en Musicología (Premio Nacional de final de licenciatura), flautista, logopeda y musicoterapeuta. Obtuvo la Mención de Doctorado Europeo en 2008, con mención de calidad, con una tesis sobre el papel de la corporeidad en la interpretación musical de instrumentos acústicos y digitales (premio extraordinario de doctorado). Ha participado en numerosos proyectos europeos, proyectos I+D+l y regionales y disfrutado más de nueve meses de estancias de investigación en centros extranjeros (Sheffield University; René Descartes-París V; InfoMus Lab [Génova]; y McGill, Music Technology Group [Montreal]). Ha sido coordinadora del Máster de Arteterapia y Educación Artística para la Inclusión Social (UVa, UCM, UAM) y es la presidenta del comité de Expertos en Musicoterapia de la asociación profesional AELFA-IF. Sus líneas de investigación están orientadas hacia el estudio de los procesos cognitivos corporeizados $4 \mathrm{E}$ en la experiencia musical y las prácticas musicales inclusivas mediadas por la tecnología, habiendo publicado más de cincuenta escritos en relación con estos temas.

ORCID: https://orcid.org/0000-0002-5725-0639

Researcher ID: AAA-2371-2019

Google Scholar: https://scholar.google.es/citations?user=PXbEm9UA AAAJ\&hl=es\&oi=ao

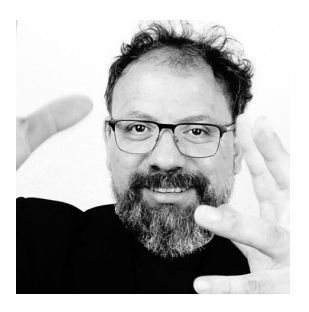

\section{Rubén López-Cano}

Escuela Superior de Música de Catalunya rlopez1@esmuc.cat

Rubén López-Cano es especialista en retórica y semiótica musicales, filosofía de la cognición corporizada de la música, música popular urbana, reciclaje musical desde la Edad Media hasta la era mashup, memes y cultura musical digital, musicología audiovisual, diáspora, cuerpo y subjetividad musicales, investigación artística y epistemología de la investigación musical.

Es autor de un centenar de artículos académicos y de los libros Música plurifocal (México: JGH, 1997), Música y retórica en el Barroco (Barcelona: Amalgama, 2012), Cómo hacer una comunicación, ponencia o paper y no morir en el intento (Barcelona: SIbE, 2012), Investigación artística en música: problemas, experiencias y propuestas (en coautoría con Úrsula San Cristóbal) (Barcelona: Fonca-Esmuc, 2014), Música dispersa. Apropiación, influencias, robos y remix en la era de la escucha digital (Barcelona: Musikeion books, 2018) y La música cuenta. Retórica, narratividad, dramaturgia, cuerpo y afectos (Barcelona: Fonca-Esmuc, 2020). 


\section{artnodes}

https://artnodes.uoc.edu

Neurodiversidad y cognición 4E. Música y multisensorialidad en los entornos Metatopia

Dirigió TRANS. Revista Transcultural de Música (2005-2013) y pertenece a los consejos asesores de diversas revistas académicas de Europa y América Latina.

ORCID ID: orcid.org/0000-0001-9266-5138

Scopus Author ID: 56481581200

Web of Science Researcher ID X-7760-2019

Google Scholar: https://scholar.google.es/citations?user=qi_ UCroAAAAJ\&hl=es\&oi=ao

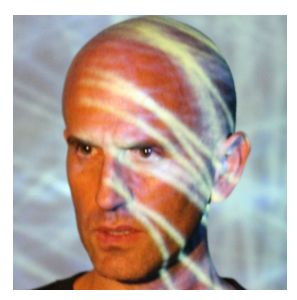

\section{Jaime del Val}

Instituto Metabody /Reverso

jaimedelval@metabody.eu

Jaime del Val es artista multidisciplinar, filósofo, promotor del Proyecto Metabody y de Reverso. Desde 2001 desarrolla una convergencia entre artes (danza, performance, artes visuales, música, arquitectura), nuevas tecnologías, pensamiento crítico (poshumanismo queer) y activismo. Sus proyectos se han presentado en más de treinta países de cuatro continentes. Desde 2013 coordina el proyecto Metabody, con una red de 38 socios de dieciséis países, habiendo organizado más de cincuenta foros internacionales y coordinado más de sesenta proyectos de investigación. Ha publicado más de ciento veinte ensayos de filosofía y pensamiento crítico en revistas como Leonardo, World Futures o Performance Research y con editoriales como MIT, Palgrave o Routledge y ha impartido más de ciento cincuenta conferencias en universidades como U. C. Berkeley, Stanford, MIT Media Lab, Yale, Duke 0 Cambridge. Cofunda en 2010 el metahumanismo, promueve numerosas actividades en el ámbito del poshumanismo crítico, y ha editado las Revistas Metabody y Reverso. Como pianista y compositor ha grabado trece discos y más de trescientas obras, y como artista plástico ha expuesto obra pictórica y fotográfica desde 1997. También desde entonces ha desarrollado un activismo continuo en numerosos movimientos, especialmente LGTB-queery ecologista, que ocasionalmente ha coordinado a nivel nacional e internacional.

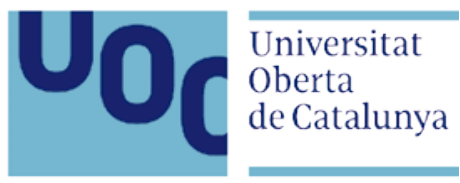

